\title{
Fast Algorithms for Power Grid Analysis Based on Effective Resistance
}

\author{
Selçuk Köse and Eby G. Friedman \\ Department of Electrical and Computer Engineering \\ University of Rochester \\ Rochester, New York 14627 \\ \{kose,friedman\}@ece.rochester.edu
}

\begin{abstract}
The size of on-chip power distribution networks is increasing with each technology generation. Accurate and computationally efficient analysis of these power distribution networks has therefore become increasingly challenging. High performance power distribution networks are generally implemented as a uniform mesh structure. The uniformity of these power distribution networks can be exploited for fast, accurate nodal analysis. A closed form expression is presented here for determining the voltage at any arbitrary node in a power distribution network. The error of the proposed method as compared with SPICE is less than $0.2 \%$. Since no iterations are required, the proposed method significantly outperforms previously proposed power grid analysis techniques in terms of computational speed while exhibiting low error.
\end{abstract}

\section{INTRODUCTION}

The design of power distribution networks is highly challenging with each technology generation. Reduced supply voltages and increased current demands have placed stringent constraints on the design of high performance power distribution networks [1]-[3]. Due to the resistive nature of the power distribution network, the supply voltage delivered to the load circuitry is lower than the supply voltage generated at the output of the power supply. This voltage difference depends upon both the characteristics of the power distribution network and the current demand of the local load circuitry. The voltage loss within the power distribution network degrades circuit performance in terms of increased delay, delay uncertainty, and signal skew [1].

Power distribution networks are generally modeled as a uniform grid structure. In a uniform grid structure, the effective impedance between any two arbitrary nodes depends upon the distance between the two nodes and the impedance of the power grid. The effective resistance between any two nodes in a uniform grid structure has been considered by Venezian in [4], where he formulated the resistance between any two nodes in an infinite resistive grid. Since the voltage drop at a node is a function of the resistance between that node and the power supply, the effective resistance considering the power supply voltage and load current characteristics supplies

This research is supported in part by the National Science Foundation under Grant Nos. CCF-0541206, CCF-0811317, and CCF-0829915, grants from the New York State Office of Science, Technology and Academic Research to the Center for Advanced Technology in Electronic Imaging Systems, and by grants from Intel Corporation, Eastman Kodak Company, and Freescale Semiconductor Corporation. sufficient information to determine the voltage drop at any particular node. In this paper, closed form expressions are provided for fast $\mathrm{P} / \mathrm{G}$ network analysis by exploiting this effective resistance concept.

$\mathrm{P} / \mathrm{G}$ distribution networks within modern integrated circuits contain millions of nodes. Since the $\mathrm{P} / \mathrm{G}$ network is a linear network, formulation of the analysis process is straightforward. The solution of this linear system, however, is infeasible due to the large grid size. If the power distribution system has $N$ rows and $N$ columns, the resulting conductance matrix to solve this power distribution network is $N^{2} \mathrm{x} N^{2}$. The size of the conductance matrix therefore increases quadratically with increasing power grid size. Due to the large size of these power distribution networks, traditional linear solvers are incapable of solving this large system in reasonable time [5]-[7].

Several methods have been proposed for efficient power grid analysis; 1) reduce the size of the linear system, 2) iteratively solve the linear system, and 3) apply advanced linear algebraic techniques to exploit the sparse nature of the power grid. Conventional interconnect model order reduction techniques [8] are applicable for tree structured interconnects; however, these structures are inappropriate for power distribution networks which are generally mesh structured. The power grid can be reduced to a simpler structure where this coarse structure is later mapped into the original grid [3]. The power grid has been optimized to minimize the $I R$ drop at the grid center in [9]. In [10], the power grid is partitioned into a number of smaller parts where each partition is analyzed separately. Random walk techniques are used to analyze the power grid in [5] to iteratively solve the $I R$ drop problem without computing large matrix operations. In [11], the power supply network is analyzed using stochastic voltage prediction. Two efficient iterative algorithms are proposed in [6] to compute the $I R$ drop within a power grid. Although these algorithms are faster than conventional linear solvers, significant computational time is required to iteratively solve these algorithms. A closed form expression would solve this problem.

Uniform current loads are generally assumed in power distribution networks to exploit symmetry in a linear system. In [12], an $I R$ drop analysis is described for a power grid structure with semi-uniform current loads (e.g., uniform load currents are assumed within each quadrant of the distribution network). To the authors' knowledge, no closed form expressions exist that describe the voltage drop at any node within a 


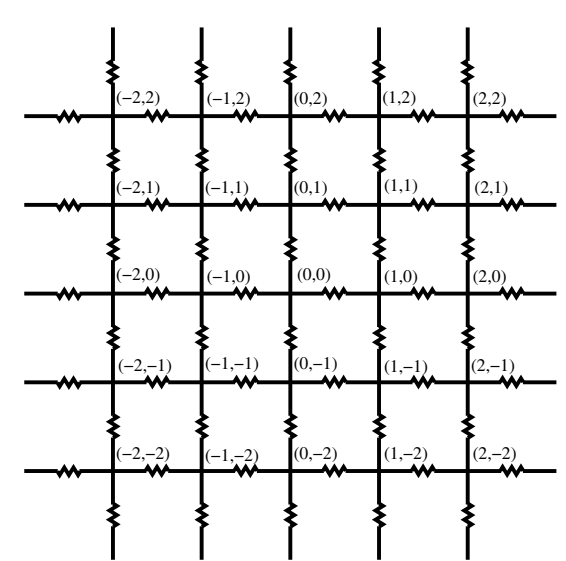

Fig. 1. Infinite resistive mesh structure to model a power distribution network.

uniform power distribution network with non-uniform current loads.

In this paper, closed form expressions for the $I R$ drop within a uniform power distribution grid with non-uniform current loads are provided. The proposed method exploits the impedance characteristics of the power distribution network between the active circuit blocks to provide these closed form expressions. Since no iterations are required to compute the proposed closed form expressions at any node, the proposed algorithm outperforms previously proposed techniques with low error.

The rest of the paper is organized as follows. The power grid model used in the analysis is described and the effective resistance concept is explained in Section II. In Section III, the proposed algorithms are reviewed. Simulation results are provided in Section IV to evaluate the validity of the proposed algorithms. Finally, the paper is concluded in Section V.

\section{BACKGROUND}

Power distribution networks are generally modeled as uniform grid structures. Due to the large size, the power grid can be modeled as an infinite number of identical resistors structured to form a square grid network. Depending upon the grid structure and the operating frequency, inductances in series with resistors and decoupling and intrinsic device capacitances can be included within the power grid model [2]. Since only the DC voltage drop is of concern in this paper, the power grid is modeled in this paper as a purely resistive grid [6], as depicted in Fig. 1. All of the resistive sections have a resistance $R$. Due to the large power grid size (i.e., tens of thousands of nodes), the grid structure is treated as infinite.

Venezian in [4] considers the effective resistance between any two arbitrary nodes within a uniform infinite grid structure by exploiting the principle of superposition. Venezian developed an exact solution for the effective resistance between any two nodes, $N_{1}\left(x_{1}, y_{1}\right)$ and $N_{2}\left(x_{2}, y_{2}\right)$, in an infinite grid as

$$
R_{m, n}=\int_{0}^{\pi} \frac{\left(2-e^{-|m| \alpha} \cos (n \beta)-e^{-|n| \alpha} \cos (m \beta)\right)}{\sinh (\alpha)} d \beta .
$$

TABLE I

VALIDITY OF THE effective resistance MODEL IN [4].

\begin{tabular}{l||l|l|l|l|l}
\hline & $\mathrm{R}_{1,0}$ & $\mathrm{R}_{1,1}$ & $\mathrm{R}_{3,4}$ & $\mathrm{R}_{5,0}$ & $\mathrm{R}_{10,10}$ \\
\hline \hline Exact solution (1) & 0.5 & 0.636 & 1.028 & 1.026 & 1.358 \\
\hline Approximation (2) & 0.515 & 0.625 & 1.027 & 1.027 & 1.358 \\
\hline Error (\%) & 3 & 1.8 & 0.1 & 0.1 & 0 \\
\hline
\end{tabular}

Venezian also provides a closed form approximation ${ }^{1}$ for (1) as

$$
R_{m, n}=\frac{1}{2 \pi} * \ln \left(n^{2}+m^{2}\right)+0.51469,
$$

where

$$
m=\left|x_{1}-x_{2}\right| \text { and } n=\left|y_{1}-y_{2}\right| .
$$

$\alpha$ and $\beta$ are used to rewrite Kirchhoff's node equations as difference equations. The interested reader is urged to read [4] for a complete explanation.

The error with approximation (2) is less than $3 \%$ as compared to the exact solution in (1). A few examples that demonstrate the validity of (2) are listed in Table I. As tabulated in Table I, the error quickly approaches zero with increasing distance between two nodes.

\section{Closed Form EXPRESSIONS FOR NODAL ANALYSiS IN A UNIFORM POWER GRID}

Two algorithms are proposed in this paper to analyze the voltage at any arbitrary node within a uniform power distribution network:

- one power supply and one current load placed arbitrarily within the distribution network.

- one power supply and multiple current loads placed arbitrarily within the distribution network.

Since the distance between two nodes does not affect the computational complexity of determining the effective resistance, the computational complexity of the proposed algorithms is independent of the size of the power grid. A primary advantage of the proposed algorithms is that these algorithms can determine the voltage at any node without determining the voltage at adjacent nodes which may not be of interest.

\section{A. One Power Supply and One Load Circuit}

In this section, the voltage at an arbitrary node Node $_{1}$, shown in Fig. 2a, is determined when one power supply and one current load exist within the power grid. The power grid model shown in Fig. 2 a reduces this system to an effective resistance model, as illustrated in Fig. 2b. The effective resistance between $\mathrm{N}_{\text {supply }}$ and Node $_{1}$, Node $\mathrm{N}_{1}$ and $\mathrm{N}_{\text {load }}$, and $\mathrm{N}_{\text {supply }}$ and $\mathrm{N}_{\text {load }}$ are denoted as $R_{s n}, R_{n l}$, and $R_{s l}$, respectively. These resistances are determined from (2). The voltage at $\mathrm{N}_{\text {load }}$ is

$$
V_{\text {load }}=V_{\text {supply }}-I_{\text {load }} * R_{\text {sl }} .
$$

After determining the voltage at $\mathrm{N}_{\text {load }}$ (see Fig. 2b), the voltage at Node $_{1}$ can be found as follows. Assume that the

\footnotetext{
${ }^{1}$ The formula has been slightly modified from the original paper to produce
} more accurate results. 


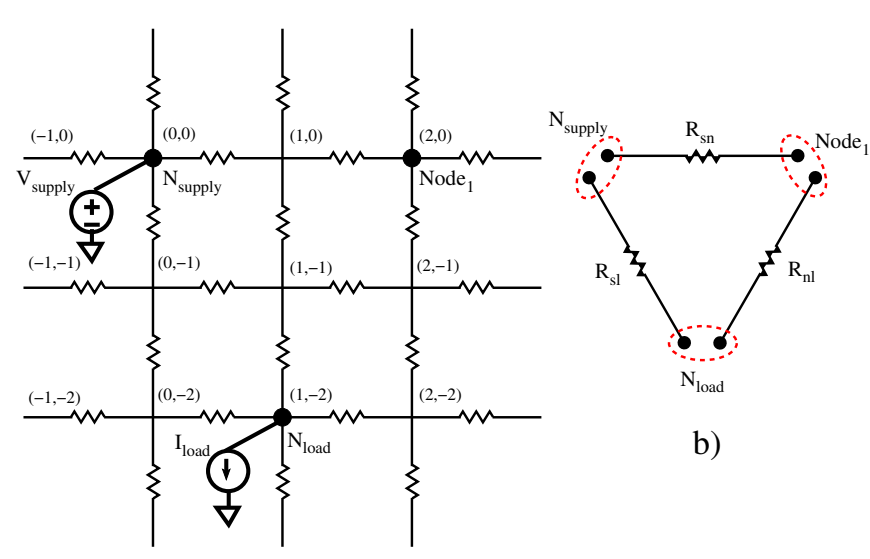

a)

Fig. 2. Power distribution grid model a) one power supply connected at $(0,0)$ and one current load connected at $(1,-2)$ and $b)$ corresponding reduced effective resistance model.

load current $I_{\text {load }}$ flows from $\mathrm{N}_{\text {supply }}$ to $\mathrm{N}_{\text {load }}$ along the path $R_{s n}-$ Node $_{1}-R_{n l}$. The voltage at Node $V_{N_{\text {ode }}}$ is

$$
V_{\text {Node }_{1}}=V_{\text {supply }}-I_{\text {load }} * R_{\text {sn }}
$$

or

$$
V_{\text {Node }_{1}}=V_{\text {load }}+I_{\text {load }} * R_{n l} .
$$

Since the voltage at Node $_{1}$ can be calculated with either (5) or (6), the arithmetic mean of the voltages found by (5) and (6) lowers the error. The voltage at Node $_{1}$ is therefore

$$
V_{\text {Node }_{1}}=\left[V_{\text {supply }}+V_{\text {load }}+I_{\text {load }} *\left(R_{n l}-R_{\text {sn }}\right)\right] / 2 .
$$

Substituting (4) into (7), the voltage at Node $_{1}$ is

$$
V_{\text {Node }_{1}}=\left[2 * V_{\text {supply }}+I_{\text {load }} *\left(R_{n l}-R_{\text {sn }}-R_{\text {sl }}\right)\right] / 2 .
$$

An algorithm to determine the voltage at any arbitrary node within a uniform power grid with one current load and one voltage supply is summarized in Fig. 3 (Algorithm I).

One Power Supply and One Current Load

1. Given: Supply voltage $\left(V_{\text {supply }}\right)$, load current $\left(I_{\text {load }}\right)$. Locations of voltage supply $\left(\mathrm{N}_{\text {supply }}\right)$, current load $\left(\mathrm{N}_{\text {load }}\right)$, and Node $_{1}$.

2. Calculate the effective resistances, (2), between

a) $\mathrm{N}_{\text {supply }}$ and Node $_{1}, R_{\text {sn }}$

b) Node $_{1}$ and $\mathrm{N}_{\text {load }}, R_{n l}$

c) $\mathrm{N}_{\text {supply }}$ and $\mathrm{N}_{\text {load }}, R_{\text {sl }}$

3. Calculate the voltage at $\mathrm{N}_{\text {load }}$, (4).

4. Calculate the voltage at $\operatorname{Node}_{1}, V_{N_{o d e}},(7)$

Fig. 3. Algorithm I. Voltage at an arbitrary node within a power grid with one power supply and one current load.

\section{B. One Power Supply and Multiple Load Circuits}

In this section, the voltage at an arbitrary node within a power distribution network is determined when one power supply and multiple current loads exist within a grid, as shown

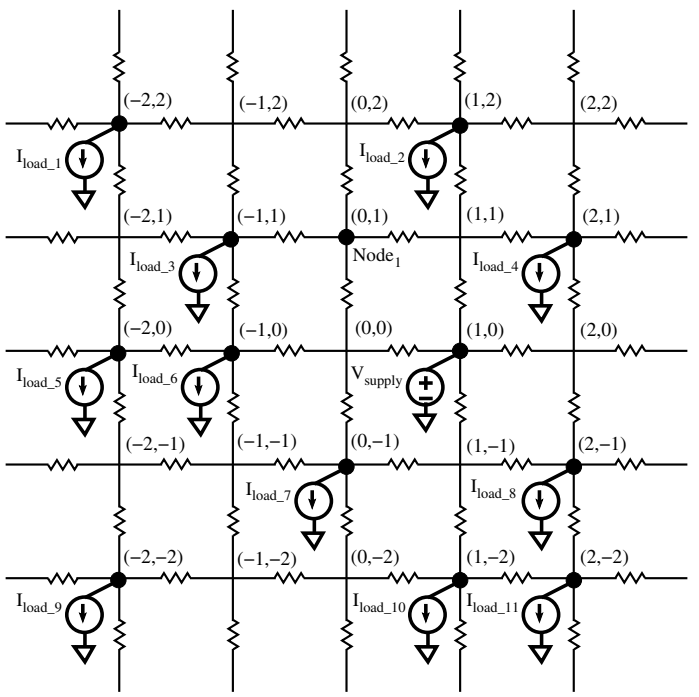

Fig. 4. Model of power distribution grid when one power supply is connected to node $(1,0)$ and multiple current loads model the load circuits connected at various nodes within the power distribution grid.

in Fig. 4. Since the current loads are assumed to be ideal current sources, the principle of superposition is applied to provide a closed form expression for the $I R$ voltage drop. Superposition is possible since linear current loads are used to model the active circuit structures. By applying superposition to each individual current load, the voltage at Node $_{1}$ is

$$
\begin{aligned}
V_{\text {Node }_{1}} & =V_{\text {supply }} \\
& -\frac{1}{2} \sum_{i=1}^{n}\left[I_{\text {load }(i)} *\left(R_{s n}+R_{s l(i)}-R_{n l(i)}\right)\right],
\end{aligned}
$$

where $n$ is the number of current loads, $I_{\text {load(i) }}$ is the $i^{\text {th }}$ current load, $R_{\text {sl(i) }}$ is the effective resistance between $\mathrm{N}_{\text {supply }}$ and the $i^{\text {th }}$ current load, and $R_{n l(i)}$ is the effective resistance between Node $_{1}$ and the $i^{\text {th }}$ current load within the power grid. An algorithm to determine the voltage at any arbitrary node with one voltage supply and multiple current loads connected to a power distribution grid is provided in Fig. 5 (Algorithm II).

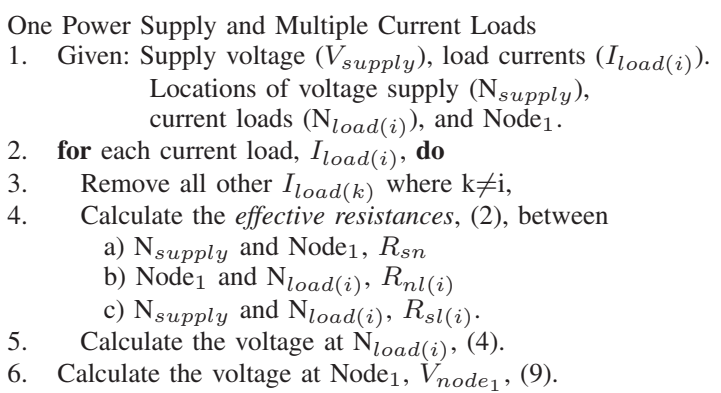

Fig. 5. Algorithm II. Voltage at arbitrary node Node $_{1}$ within a power grid with one power supply and multiple current loads, as shown in Fig 4. 
TABLE II

Error of Algorithm I AS COMPAREd to SPICE. The Voltage Supply is CONNECTED AT N3,3 (THE Light-GREy BoX) AND THE LOAD Device is Connected at $\mathrm{N}_{5,4}$ (The Dark-Grey BoX). The Maximum ERror is Less Than 0.2\% of THE Supply Voltage.

\begin{tabular}{c||c|c|c|c|c|c|c|c}
\hline & 1 & 2 & 3 & 4 & 5 & 6 & 7 & 8 \\
\hline \hline 1 & -0.12 & -0.05 & 0.46 & -0.27 & -0.49 & -0.26 & -0.125 & -0.15 \\
\hline 2 & -0.09 & -0.55 & 0.79 & 0.152 & -0.68 & -0.37 & -0.554 & -0.14 \\
\hline 3 & 0.33 & 0.62 & 0 & 1.13 & -0.52 & 0.52 & 0 & -0.26 \\
\hline 4 & -0.31 & -0.83 & 0.21 & -1.44 & -0.31 & -0.93 & -0.64 & -0.41 \\
\hline 5 & -0.25 & -0.27 & 0.37 & 0.24 & -1.10 & 0.24 & -0.22 & -0.38 \\
\hline 6 & -0.18 & -0.04 & 0.18 & -0.04 & -0.77 & -0.25 & -0.18 & -0.30 \\
\hline 7 & -0.13 & -0.01 & 0 & -0.23 & -0.50 & -0.36 & -0.28 & -0.30 \\
\hline 8 & -0.14 & -0.04 & -0.08 & -0.27 & -0.32 & -0.34 & -0.34 & -0.34 \\
\hline
\end{tabular}

\section{EXPERIMENTAL RESULTS}

The validity of the proposed nodal voltage analysis in a uniform power distribution grid is presented in this section. The resistance between adjacent nodes is $1 \Omega$ and the supply voltage is 1 volt. The active devices are modeled as ideal current loads. The current loads and the voltage supplies are arbitrarily placed within the uniform power grid.

The validity of the proposed closed form expression for one voltage supply and one current load is analyzed with a 1 volt supply connected at $\mathrm{N}_{3,3}$ and the load sinking $100 \mathrm{~mA}$ at $\mathrm{N}_{5,4}$. The maximum error is $1.44 \mathrm{mV}$, less than $0.2 \%$ of the supply voltage. The error of the corresponding node voltages as compared to SPICE is listed in Table II. The light-grey box is the supply node and the dark-grey box is the node where the current load is connected.

The nodal voltage analysis of a power grid with one voltage supply and multiple current loads is evaluated when the voltage supply is connected to $\mathrm{N}_{4,4}$ and four current loads are randomly placed at $\mathrm{N}_{1,7}, \mathrm{~N}_{2,3}, \mathrm{~N}_{6,6}$, and $\mathrm{N}_{2,7}$. In this case, each load sinks $25 \mathrm{~mA}$ from the power grid. The error of Algorithm II as compared to SPICE for a $50 \times 50$ uniform power grid is listed in Table III. The maximum error of the proposed algorithm as compared to SPICE is $1.1 \mathrm{mV}$ (less than $0.2 \%$ ).

\section{CONCLusions}

Closed form expressions for the analysis of large uniform power grids are provided in this paper. Two algorithms are described for fast and accurate power grid analysis of modern high complexity integrated circuits. Algorithm I determines the voltage drop with one power supply and one current load arbitrarily placed within the distribution grid. Algorithm II is used for nodal analysis when one power supply and multiple current loads exist within the power grid. The maximum error of both algorithms is less than $0.2 \%$ of the power supply voltage. Since the proposed algorithms use closed form expressions rather than iteratively determining the voltage, the computational runtime is significantly smaller than previously proposed power grid analysis methods while exhibiting low
TABLE III

Error of Algorithm II AS Compared to SPICE. The Voltage Supply is Connected at $\mathrm{N}_{4,4}$ (THE Light-GRey BoX) AND THE LOAD Devices ARe Connected at $\mathrm{N}_{1,7}, \mathrm{~N}_{2,3}, \mathrm{~N}_{6,6}$, AND $\mathrm{N}_{2,7}$ (THE DARK-Grey BoXes). The MAXimum ERror IS LesS Than $0.2 \%$ OF THE Supply Voltage.

\begin{tabular}{c||c|c|c|c|c|c|c|c}
\hline & 1 & 2 & 3 & 4 & 5 & 6 & 7 & 8 \\
\hline \hline 1 & -0.17 & -0.24 & -0.06 & 0.07 & -0.16 & -0.38 & -0.19 & -0.30 \\
\hline 2 & 0.01 & -0.40 & -0.06 & 0.32 & -0.20 & -0.14 & -0.36 & -0.03 \\
\hline 3 & -0.23 & -0.25 & -0.80 & 0.60 & -0.64 & -0.25 & -0.18 & -0.02 \\
\hline 4 & 0.28 & 0.11 & 0.76 & 0 & 0.69 & 0.18 & 0.05 & 0.02 \\
\hline 5 & 0.01 & -0.40 & -0.64 & 0.75 & -0.50 & -0.42 & -0.06 & -0.04 \\
\hline 6 & 0 & -0.49 & -0.08 & 0.28 & -0.39 & -0.31 & -0.36 & -0.23 \\
\hline 7 & -0.40 & -0.25 & -0.32 & 0.12 & -0.04 & -0.45 & -0.07 & -0.13 \\
\hline 8 & -0.05 & -0.35 & 1.11 & 0.08 & -0.02 & -0.29 & -0.16 & -0.17 \\
\hline
\end{tabular}

error. Another advantage is that the proposed methods compute the voltage at any node within a power grid without determining the voltage at adjacent nodes. The proposed algorithm can therefore be applied to localized power grid analysis.

\section{REFERENCES}

[1] M. Popovich, A. V. Mezhiba, and E. G. Friedman, Power Distribution Networks with On-Chip Decoupling Capacitors, Springer, 2008.

[2] S. Pant, D. Blaauw, and E. Chiprout, "Power Grid Physics and Implications for CAD," IEEE Design and Test of Computers, Vol. 24, No. 3, pp.246-254, May 2007.

[3] J. N. Kozhaya, S. R. Nassif, and F. N. Najm, "A Multigrid-Like Technique for Power Grid Analysis," IEEE Transactions on ComputerAided Design of Integrated Circuits and Systems, Vol. 21, No. 10, pp. 1148-1160, October 2002.

[4] G. Venezian, "On the Resistance between Two Points on a Grid," American Journal of Physics, Vol. 62, No. 11, pp. 1000-1004, November 1994.

[5] H. Qian, S. R. Nassif, and S. S. Sapatnekar, "Random Walks in a Supply Network," Proceedings of the IEEE/ACM Design Automation Conference, pp. 93-98, June 2003.

[6] Y. Zhong and M. D. F. Wong, "Fast Algorithms for IR Drop Analysis in Large Power Grid," Proceedings of the IEEE/ACM International Conference on Computer-Aided Design, pp. 351-357, November 2005.

[7] Y. Zhong and M. D. F. Wong, "Efficient Second-Order Iterative Methods for IR Drop Analysis in Power Grid," Proceedings of the IEEE/ACM Design Automation Conference, pp. 768-773, January 2007.

[8] Y. I. Ismail and E. G. Friedman, "DDT: Direct Derivation of Transfer Function-An Alternative to Moment Matching for Tree Structured Interconnect," IEEE Transactions on Computer-Aided Design of Integrated Circuits and Systems, Vol. 21, No. 2, pp. 131-144, February 2002.

[9] D. E. Khalil and Y. Ismail, "Optimum Sizing of Power Grids for IR Drop," Proceedings of the IEEE International Symposium of Circuits and Systems, pp. 481-484, May 2006.

[10] H. Li et al., "Partitioning-Based Approach to Fast On-Chip Decap Budgeting and Minimization," IEEE Transactions on Computer-Aided Design of Integrated Circuits and Systems, Vol. 25, No. 11, pp. 24022412, November 2006.

[11] A. B. Kahng, B. Liu, and Q. Wang, "Stochastic Power/Ground Supply Voltage Prediction and Optimization Via Analytical Placement," IEEE Transactions on Very Large Scale Integration (VLSI) Systems, Vol. 15, No. 8, pp. 904-912, August 2007.

[12] P. Gupta and A. B. Kahng, "Efficient Design and Analysis of Robust Power Distribution Meshes," Proceedings of the IEEE International Conference on VLSI Design, pp. 337-342, January 2006. 